\title{
Axisymmetric Finite Element Solution of Non-isothermal Parallel-plate Flow
}

\author{
Shangyou Zhang and David O. Olagunju* \\ Department of Mathematical Sciences \\ University of Delaware \\ Newark, DE 19716. USA
}

November 19, 2004

\begin{abstract}
Steady non-isothermal parallel-plate flow of a Newtonian fluid with a temperature dependent viscosity is considered. The viscosity is modelled by a Nahme type law. We apply axisymmetric $Q^{k}$ finite elements to the coupled nonlinear system to obtain numerical solutions for a wide range of parameters.
\end{abstract}

Keywords: Parallel-plate flow, viscous heating, axisymmetric finite elements.

AMS subject classification: 76D07, 76M10,65F10

*corresponding author: olagunju@math.udel.edu 


\section{Introduction}

It is a well known fact that heating effects due to viscous dissipation could cause appreciable deviation from isothermal theory in shear flow. If not properly accounted for, this could lead to errors in viscometric applications $[1,2,3,4,5]$. Viscous heating has also been shown to significantly alter the onset and nature of instabilities in shear flows $[7,8,9]$.

Due to the strong coupling between the velocity and temperature fields, no exact solution of the non-isothermal flow between finite coaxial parallel plates exist. Consequently, approximate techniques have been used to reduce the equations to more tractable forms. For example, in [2] Bird and Turian assumed a velocity profile of the form $v_{\theta}=r g(z)$, which satisfies the momentum equation only if the flow is isothermal. Regular perturbation methods have also been used. If the aspect ratio $\alpha \equiv H / a$, where $a$ is the disk radius and $H$ is the gap, an exact solution exists for the limiting case $\alpha=0[3,6,10]$. In this limit the problem reduces to that of plane Couette flow. However, the plane Couette solution does not satisfy the boundary conditions at the fluidair interface since the problem is singular in the limit $\alpha \rightarrow 0$. In [3], Turian and Bird noted that errors up to $15 \%$ existed between the theoretical results based on the plane Couette solution and experiments. They suggested that this discrepancy could be due to edge effects among other probable causes. In [11], Olagunju considered edge effects in non-isothermal parallel-plate flow in the case when the Nahme-Griffith number $N a$ is small. In the present study a finite element method is used to solve the full coupled temperature and velocity equations in a bounded region for a wide range of the aspect ratio and the Nahme-Griffith number. We then compare the results with the approximate solutions obtained elsewhere.

\section{Governing Equations}

We consider steady, axisymmetric, non-isothermal creeping flow of an incompressible Newtonian fluid. The analysis will be given in detail for flow between two coaxial disks of radius $a$, separation $H$, and aspect ratio $\alpha \equiv H / a$. Similar equations can be obtained for a cone-and-plate system with gap angle $\alpha$. The fluid is subjected to a shearing motion by rotating the top plate at a constant angular speed $\omega$.

In dimensional form, the equation governing the steady creeping flow of an incompressible viscoelastic fluid are the continuity equation

$$
\nabla \cdot \mathbf{v}=0
$$

and the momentum equation

$$
0=-\nabla p+\nabla \cdot(\mu \dot{\gamma}),
$$

where the rate of strain tensor is defined by $\dot{\gamma}=\nabla \mathbf{v}+(\nabla \mathbf{v})^{T}$. The energy equation for the temperature $T$ is

$$
\rho c_{p} \mathbf{v} \cdot \nabla T=\kappa \nabla^{2} T+\mu \dot{\gamma}: \nabla \mathbf{v}
$$

where $c_{p}$ is the specific heat and $\kappa$ is the thermal conductivity which is assumed to be constant. In a cylindrical coordinate system $(r, \theta, z)$, we seek an axisymmetric steady solution of the form

$$
\mathbf{v}=(0, W, 0)
$$


The equations for the azimuthal velocity $W$ and a reduced temperature $\Theta$ are

$$
\frac{\partial W}{\partial z^{2}}+\alpha^{2}\left(\frac{\partial W}{\partial r^{2}}+\frac{1}{r} \frac{\partial W}{\partial r}-\frac{W}{r^{2}}\right)=\frac{\partial \Theta}{\partial z} \frac{\partial W}{\partial z}+\alpha^{2} \frac{\partial \Theta}{\partial r}\left(\frac{\partial W}{\partial r}-\frac{W}{r}\right),
$$

and

$$
\begin{aligned}
& \frac{\partial \Theta}{\partial z^{2}}+\alpha^{2}\left(\frac{\partial \Theta}{\partial r^{2}}+\frac{1}{r} \frac{\partial \Theta}{\partial r}\right)= \\
&-\mathrm{Na} e^{-\Theta}\left[\left(\frac{\partial W}{\partial z}\right)^{2}+\alpha^{2}\left(\frac{\partial W}{\partial r}-\frac{W}{r}\right)^{2}\right],
\end{aligned}
$$

subject to the following boundary conditions

$$
\begin{aligned}
& W=0, \quad \Theta=\vartheta_{w}, \quad \text { on } z=0, \\
& W_{0}=r, \quad \Theta_{0}=\vartheta_{w} \quad \text { on } z=1, \\
& \frac{\partial W}{\partial r}-\frac{W}{r}=0, \quad \Theta=\vartheta_{w}, \quad \text { on } r=1,
\end{aligned}
$$

and

$$
W=0, \quad|\Theta|<\infty, \quad \text { at } r=0 .
$$

The boundary conditions are no slip at the solid walls, the balance of stresses at the fluidair interface, and constant temperature at the boundary. In the equations above, we have nondimensionalized radial length by the plate radius $a$, and axial length by the gap $H$. The azimuthal component of velocity has been made dimensionless by $a \omega$, pressure by $\mu_{0} \omega$, and temperature by a reference temperature $T_{0}$. The quantity $\mu_{0}$ is the viscosity at the reference temperature while the variable $\Theta$ is related to the dimensionless temperature $T$ by

$$
\Theta=\delta(T-1)
$$

Here $\delta$ is the dimensionless thermal sensitivity which is usually large so that even small variations in $T$ lead to $O(1)$ changes in $\Theta$. Due to viscous heating, the viscosity is strongly temperature dependent and is modelled by a Nahme law as $[5,6]$

$$
\mu=\mu_{0} e^{-\Theta} \text {. }
$$

The parameter in equation (2.6) is the Nahme-Griffith number at the reference temperature $\mathrm{Na}=$ $\mu_{0} \delta a^{2} \omega^{2} /\left(\kappa T_{0}\right)$.

\section{$3 \quad$ Finite elements method}

The domain $\Omega$ for numerical computation is $0<z<1$ and $0<r<1$, shown in Figure 1 . In order to apply the finite element method, we need to rewrite $(2.5)-(2.6)$ in the weak, variational form. We multiply equation (2.5) by $r V(r, z)$, the test function with boundary conditions specified in Figure 1, then we apply the integration by parts to obtain

$$
\begin{aligned}
\int_{\Omega}\left(\frac{\partial W}{\partial z} \frac{\partial V}{\partial z}+\alpha^{2} \frac{\partial W}{\partial r} \frac{\partial V}{\partial r}\right. & \left.+\alpha^{2} \frac{W V}{r^{2}}\right) r d r d z-\int_{r=1} \alpha^{2} W V d z \\
& =\int_{\Omega}\left(-\frac{\partial \Theta}{\partial z} \frac{\partial W}{\partial z}-\alpha^{2} \frac{\partial \Theta}{\partial r}\left(\frac{\partial W}{\partial r}-\frac{W}{r}\right)\right) V r d r d z .
\end{aligned}
$$


We do the same for equation (2.6) with a testing function $r V(r, z)$, but of different boundary conditions shown in Figure 1, to get

$$
\begin{aligned}
\int_{\Omega}\left(\frac{\partial \Theta}{\partial z} \frac{\partial V}{\partial z}+\right. & \left.\alpha^{2} \frac{\partial \Theta}{\partial r} \frac{\partial V}{\partial r}\right) r d r d z \\
& =N a \int_{\Omega} e^{-\Theta}\left(\left(\frac{\partial W}{\partial z}\right)^{2}+\alpha^{2}\left(\frac{\partial W}{\partial r}-\frac{W}{r}\right)^{2}\right) V r d r d z
\end{aligned}
$$

To get more accurate numerical solutions, we use the following decompositions

$$
\begin{gathered}
W=W^{b}+W^{0}=r z+W^{0}, \\
\Theta=\Theta^{b}+\Theta^{0}=\vartheta_{w}+\Theta^{0} .
\end{gathered}
$$

We seek solutions $W^{0}$ and $\Theta^{0}$ instead, which have homogeneous boundary conditions depicted in Figure 1, i.e., we will find finite element solutions $W_{h}^{0}$ and $\Theta_{h}^{0}$ where $h$ stands for the grid size. To discretize (3.1) and (3.2), due to the special domain of the unit square, one may use spectral
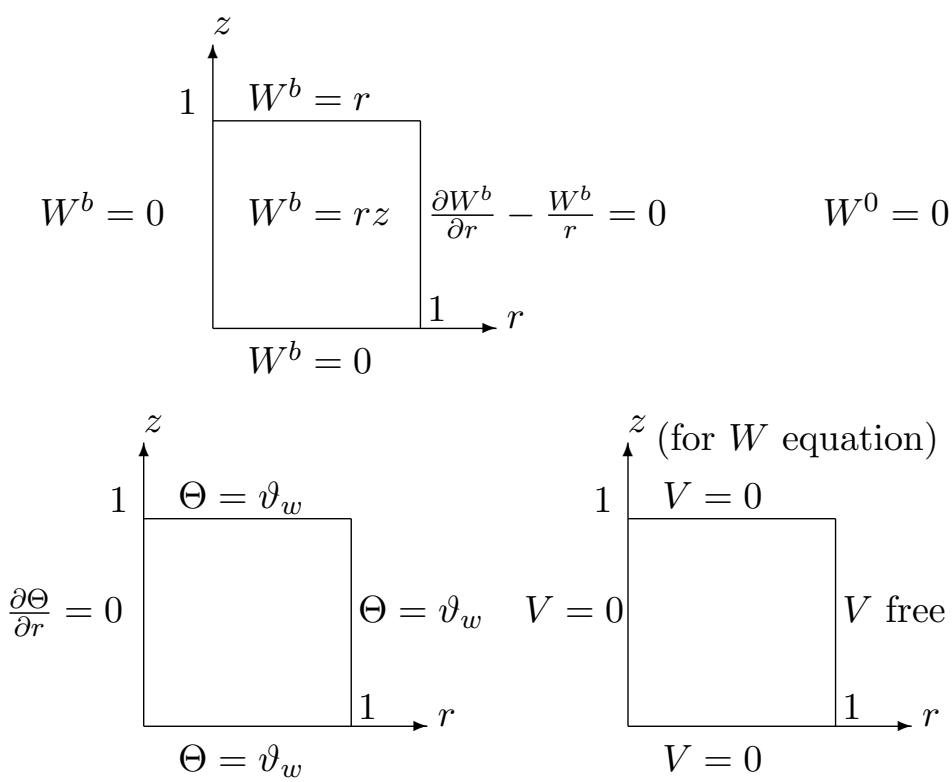
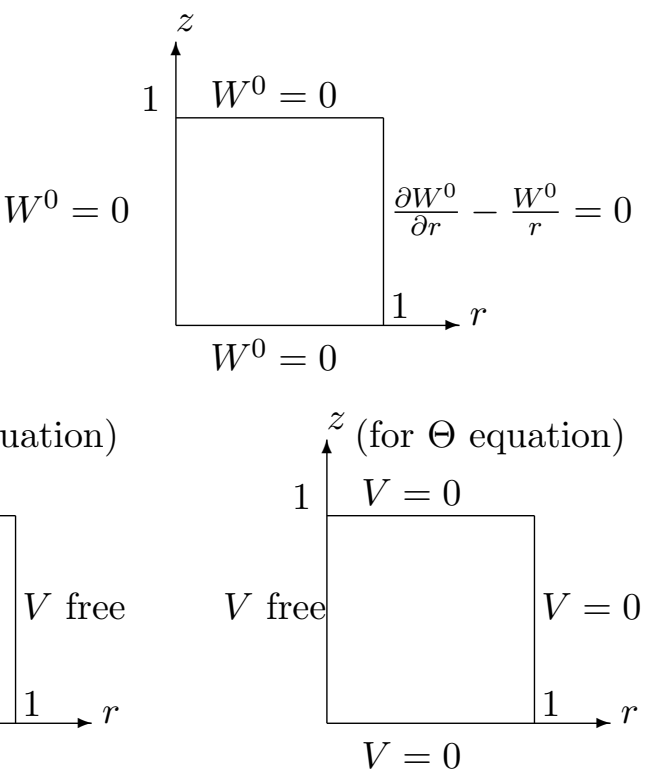

Figure 1: Boundary conditions for $W=W^{b}+W^{0}, \Theta$, and $V$ (for $W$ or $\Theta$ equation).

methods (cf. [12] and references therein) or tensor product methods (cf. [13]) to get a high order approximation. Well, the former is a special case of the latter in a general sense. To handle the nonlinearity of the coupled system, and to handle possible irregular domain in future, we use $Q^{k}$ finite elements, continuous and piecewise polynomials of separate degree $k$ or less, on uniform grids $\mathcal{K}_{h}=\left\{K \mid K=\left[r_{i}, r_{i}+h\right] \times\left[z_{j}, z_{j}+h\right]\right\}$ of $\Omega$ :

$$
\mathcal{Q}_{h}:=\left\{V \in C(\Omega)|V|_{K}=\sum_{0 \leq i+j \leq k} a_{i j} r^{i} z^{j}, \forall K \in \mathcal{K}_{h}\right\} \subset H^{1}(\Omega) .
$$


We use the following notations for the discrete spaces with homogeneous boundary conditions:

$$
\begin{aligned}
\mathcal{Q}_{h, W} & :=\mathcal{Q}_{h} \cap\{V=V(r, z) \in C(\Omega) \mid V(0, z)=V(r, 0)=V(r, 1)=0\}, \\
\mathcal{Q}_{h, \Theta} & :=\mathcal{Q}_{h} \cap\{V=V(r, z) \in C(\Omega) \mid V(1, z)=V(r, 0)=V(r, 1)=0\} .
\end{aligned}
$$

The finite element discretizations of (3.1)-(3.2) read: Find $\left(W_{h}^{0}, \Theta_{h}^{0}\right) \in \mathcal{Q}_{h, W} \times \mathcal{Q}_{h, \Theta}$ such that

$$
\begin{aligned}
a\left(W_{h}^{0}, V\right) & +\left(\frac{\alpha^{2}}{r^{2}} W_{h}^{0}, V\right)_{r}-c\left(\alpha^{2} W_{h}^{0}, V\right)=\left(-\frac{\partial \Theta}{\partial z} \frac{\partial W}{\partial z}-\alpha^{2} \frac{\partial \Theta}{\partial r}\left(\frac{\partial W}{\partial r}-\frac{W}{r}\right), V\right)_{r} \\
& -a\left(W^{b}, V\right)-\left(\frac{\alpha^{2}}{r^{2}} W^{b}, V\right)_{r}+c\left(\alpha^{2} W^{b}, V\right) \quad \forall V \in \mathcal{Q}_{h, W} \\
a\left(\Theta_{h}^{0}, V\right) & =\left(N a e^{-\Theta}\left(\left(\frac{\partial W}{\partial z}\right)^{2}+\alpha^{2}\left(\frac{\partial W}{\partial r}-\frac{W}{r}\right)^{2}\right), V\right)_{r}-a\left(\Theta^{b}, V\right) \quad \forall V \in \mathcal{Q}_{h, \Theta},
\end{aligned}
$$

where the bilinear forms are defined by

$$
\begin{aligned}
& a(U, V)=\int_{\Omega}\left(\frac{\partial U}{\partial z} \frac{\partial V}{\partial z}+\alpha^{2} \frac{\partial U}{\partial r} \frac{\partial V}{\partial r}\right) r d r d z, \\
& (U, V)_{r}=\int_{\Omega} U V r d r d z, \\
& c(U, V)=\int_{r=1,0 \leq z \leq 1} U V d z .
\end{aligned}
$$

It is not obvious how to apply the (quasi-) Newton's method to solve the nonlinear system (3.7)-(3.8) of finite element equations. The main concern is the loss of symmetry and positivity when inverting the Jacobian matrix in the Newton's method. We would solve the system by a straightforward Seidel iteration, where we solve (iteratively too) two linear systems of elliptic (see two lemmas below) finite element equations in each iteration. That is, given initially some guesses of $W_{h}^{0}$ and $\Theta_{h}^{0}$ (both are zero in real computation), we solve (3.7) to get a new $W_{h}^{0}$, where the old $W_{h}^{0}$ and $\Theta_{h}^{0}$ are used to generate the right hand side vector only. Then the new $W_{h}^{0}$ and the old $\Theta_{h}^{0}$ would be used to generate the right hand side vector in (3.8). We solve (3.8) to get a new $\Theta_{h}^{0}$. The next two lemmas show that the linear systems at each step has a unique solution, because both the coefficient matrices are symmetric and positive definite.

Lemma 3.1 For any $V \in \mathcal{Q}_{h, \Theta}$ and $V \neq 0$,

$$
a(V, V)>0 .
$$

Proof. First, the bilinear form $a(\cdot, \cdot)$ is nonnegative. If $a(V, V)=\int_{\Omega}\left[\left(\partial_{z} V\right)^{2}+\alpha^{2}\left(\partial_{r} V\right)^{2}\right] r d r d z=0$, then $\partial_{z} V=0$ almost everywhere in $\Omega$. Since $V=0$ at $z=0, V=0$ in $\Omega$.

Lemma 3.2 For any $V \in \mathcal{Q}_{h, W}$ and $V \neq 0$,

$$
a(V, V)+\left(\frac{\alpha^{2}}{r^{2}} V, V\right)_{r}-c\left(\alpha^{2} V, V\right)>0 .
$$


Proof. For any $V \in \mathcal{Q}_{h, W}$, using the integration by parts formula, we have

$$
\int_{0}^{1} \frac{\partial V}{\partial r}(r, t) V(r, t) d r=V(1, t)^{2}-V(0, t)^{2}-\int_{0}^{1} \frac{\partial V}{\partial r}(r, t) V(r, t) d r
$$

and, since $V(0, t)=0$,

$$
V(1, t)^{2}=2 \int_{0}^{1} \frac{\partial V}{\partial r}(r, t) V(r, t) d r
$$

Next, using Schwartz's inequality we obtain

$$
\begin{aligned}
V(1, t)^{2} & =\int_{0}^{1} \frac{\partial V}{\partial r}(r, t) V(r, t) d r \\
& \leq\left(\int_{0}^{1}\left(\frac{\partial V}{\partial r}(r, t)\right)^{2} r d r\right)^{1 / 2}\left(\int_{0}^{1} V(r, t)^{2} \frac{1}{r} d r\right)^{1 / 2} \\
& \leq \frac{1}{2} \int_{0}^{1}\left(\frac{\partial V}{\partial r}(r, t)\right)^{2} r d r+\frac{1}{2} \int_{0}^{1} V(r, t)^{2} \frac{1}{r} d r .
\end{aligned}
$$

Integrating both sides for $0 \leq z \leq 1$, it follows that

$$
\int_{r=1,0 \leq z \leq 1} V^{2} d z \leq \frac{1}{2} \int_{\Omega}\left(\frac{\partial V}{\partial r}\right)^{2} r d r d z+\frac{1}{2} \int_{\Omega} \frac{V^{2}}{r} d r d z
$$

Therefore, by (3.9)-(3.11)

$$
a(V, V)+\left(\frac{\alpha^{2}}{r^{2}} V, V\right)_{r}-c\left(\alpha^{2} V, V\right) \geq \int_{\Omega}\left(\left(\partial_{z} V\right)^{2}+\frac{\alpha^{2}}{2}\left(\partial_{r} V\right)^{2}+\frac{1}{2} \frac{V^{2}}{r^{2}}\right) r d r d z .
$$

Repeating the proof of Lemma 3.1, the positivity of the combined bilinear form is shown.

We remark that one may move the negative term $-c\left(\alpha^{2} W_{h}^{0}, V\right)$ in the equation (3.7) to the right hand side and compute it by previous $W_{h}^{0}$ in the iterative procedure. However, we believe that this may cause instability as the newly computed solution $W_{h}^{0}$ fails to satisfy the boundary condition at $r=1$ :

$$
\frac{\partial W_{h}^{0}}{\partial r}-\frac{W_{h}^{0}}{r}=0 \text { weakly. }
$$

We remark that, different from the finite elements in polar coordinates discretizing the 2D Laplace operator (cf. [14]), the 3D axisymmetric finite element equations for the Laplace operator is regular, measured by weighted $L_{r}^{2}(\Omega)$ (with inner product $(\cdot, \cdot)_{r}$ defined in (3.10)) and weighted $H_{r}^{k}(\Omega)$ (cf. [15]). Therefore, if we apply the multigrid method to solve (3.8), the number of arithmetic operations to solve the linear system of equations up to the order of truncation error is proportional to the number of unknowns in the system, i.e., solving a system of $N$ unknowns requires $C N$ operations when $N \rightarrow \infty$. When $N$ is large, no method can beat the optimal order multigrid method (cf. [16]). When $N$ is moderately large, say, a few hundreds or a few thousands of unknowns, the conjugate gradient iteration is usually the fastest. We adopt the conjugate gradient method for solving both (3.7) and (3.8). The other reason is that, unlike (3.8), there is a (weekly) singular $1 / r$ in the integral in (3.7). It is not known yet how to improve the fine-level smoothing iteration in the multigrid method to handle large entries of the coefficient matrix near the line $r=0$, so that the multigrid method retains its uniform contraction property. 


\section{Numerical solution}

To simplify notations further, we introduce the following bilinear form and functionals (cf. (3.9$(3.11))$.

$$
\begin{aligned}
A(U, V) & =a(U, V)+\alpha^{2}\left(\frac{U}{r}, \frac{V}{r}\right)_{r}-\alpha^{2} c(U, V) \\
F_{W, \Theta}(V) & =\left(-\frac{\partial \Theta}{\partial z} \frac{\partial W}{\partial z}-\alpha^{2} \frac{\partial \Theta}{\partial r}\left(\frac{\partial W}{\partial r}-\frac{W}{r}\right), V\right)_{r}, \\
G_{W, \Theta}(V) & =N a\left(e^{-\Theta}\left[\left(\frac{\partial W}{\partial z}\right)^{2}+\alpha^{2}\left(\frac{\partial W}{\partial r}-\frac{W}{r}\right)^{2}\right], V\right)_{r} .
\end{aligned}
$$

With new notations, we rewrite (3.7)-(3.8) as

$$
\begin{aligned}
A\left(W_{h}^{0}, V\right) & =F_{W, \Theta}(V)-A\left(W^{b}, V\right) & & \forall V \in \mathcal{Q}_{h, W}, \\
a\left(\Theta_{h}^{0}, V\right) & =G_{W, \Theta}(V)-a\left(\Theta^{b}, V\right) & & \forall V \in \mathcal{Q}_{h, \Theta} .
\end{aligned}
$$

Algorithm 4.1 The coupled nonlinear system (4.4)-(4.5) is solved by the Seidel iteration with the given initial guess $W_{h, 0}^{0}=0$ and $W_{\Theta, 0}^{0}=0$. For $j=1,2, \ldots$,

$$
W_{h, j}^{0}=W_{h, j-1}^{0}+e,
$$

where $e$ solves the equation

$$
A(e, V)=F_{W_{j-1}, \Theta_{j-1}}(V)-A\left(W^{b}, V\right)-A\left(W_{h, j-1}^{0}, V\right) \quad \forall V \in \mathcal{Q}_{h, W},
$$

and $\Theta_{h, j}^{0}$ is defined by the equation

$$
a\left(\Theta_{h, j}^{0}, V\right)=G_{W_{j}, \Theta_{j-1}}(V)-a\left(\Theta^{b}, V\right) \quad \forall V \in \mathcal{Q}_{h, \Theta} .
$$

Here $W_{j}=W^{b}+W_{h, j}^{0}$ and $\Theta_{j}=\Theta^{b}+\Theta_{h, j}^{0}$ for $j=0,1,2, \ldots$ 

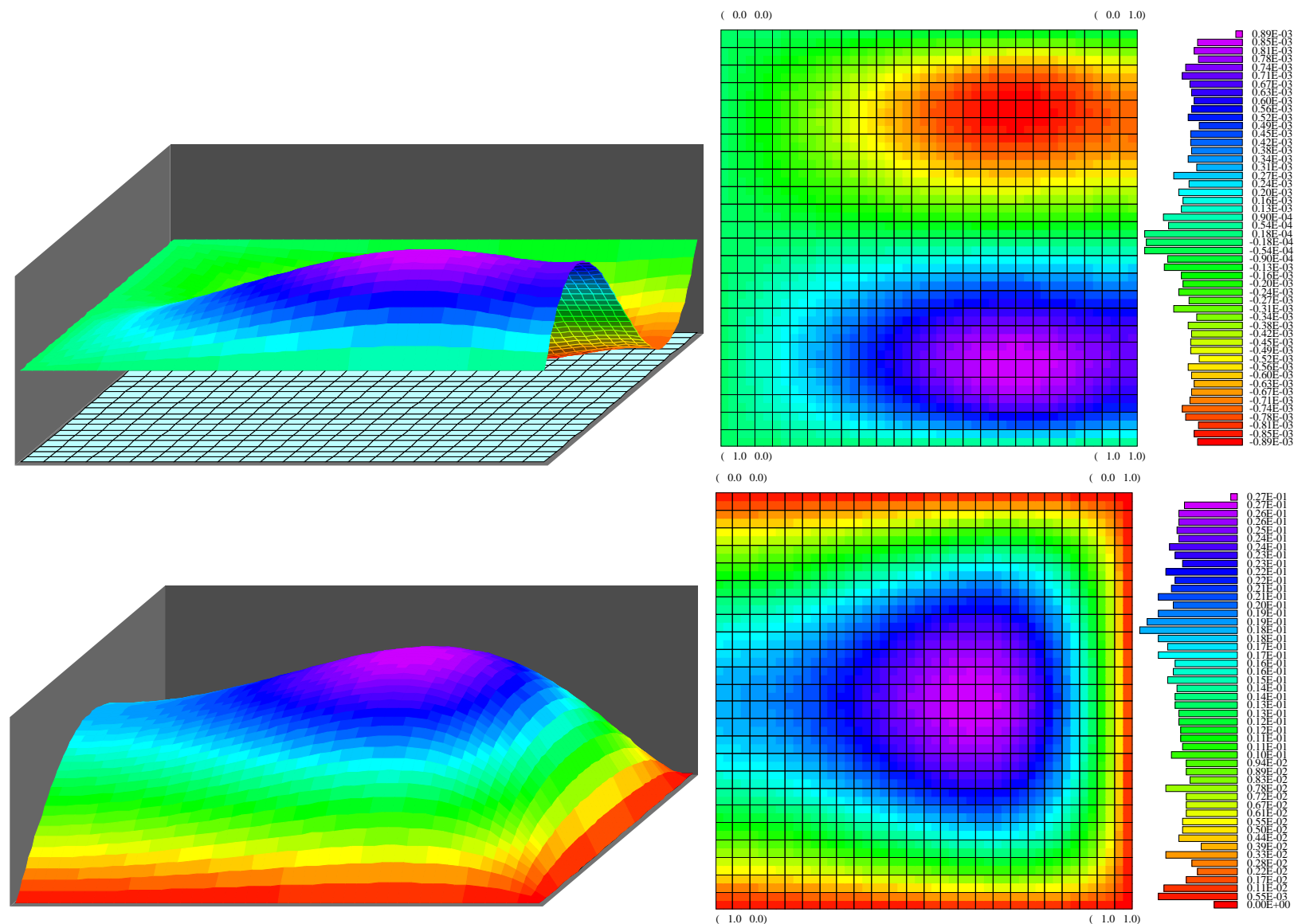

Figure 2: Numerical solutions for $W_{h}^{0}$ (left) and $\Theta_{h}$ (right), $\alpha=1$.

In Figure 2, we plotted the numerical solutions for $W$ (left) and $\Theta$ (right) with parameters

$$
\alpha=1, \quad N a=1, \quad \text { and } a_{0}=1 .
$$

Here the computation ends when the nodal differences between $W_{j}$ and $W_{j-1}$, and between $\Theta_{j}$ and $\Theta_{j-1}$, are less than $10^{-5}$. The grid size $h=1 / 4$ (the unit square is refined to level 3) and the polynomial degree $k=6$. There are 16 elements, and 49 degrees of freedom each element. The total number of unknowns in the discrete system of equations is 2401. The number of unknowns is relatively small as we used higher order finite elements. This is part of the reason that the conjugate gradient method outperforms the multigrid method. The Seidel iteration finished with 4 steps in this case. For relative large $\alpha$, the iteration converges fast. Figure 3 shows the case when $\alpha=0.1$ where we have boundary layers near the line $r=1$. There the number of Seidel iterations is 7 . 

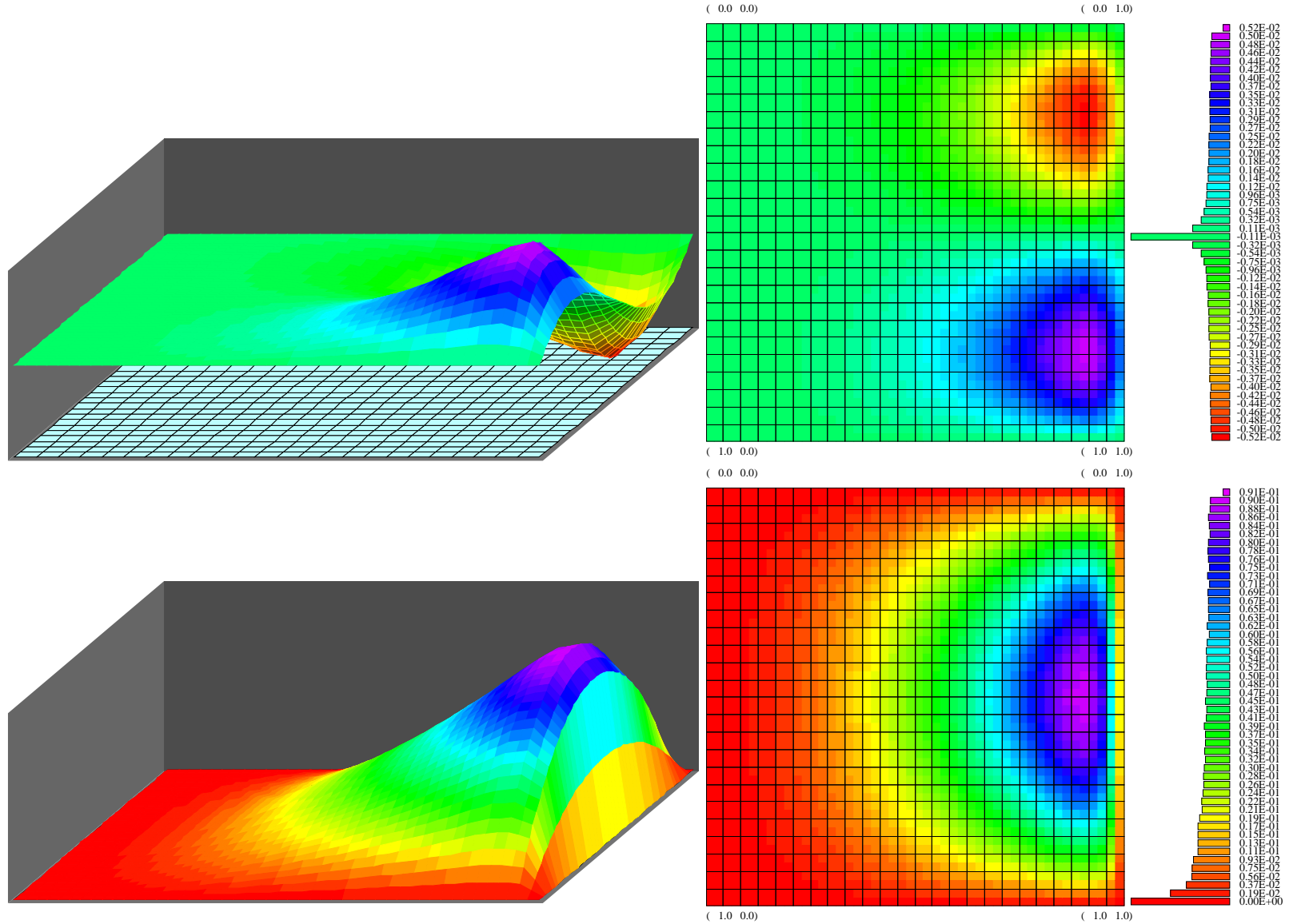

Figure 3: Numerical solutions for $W_{h}^{0}$ (top) and $\Theta_{h}$ (bottom), $\alpha=0.1$.

We note that for such a high order of finite elements, the numerical quadratures for the integrals need to be accurate. We used the product Gauss-Legendre quadratures of high enough orders for all integrals except the singularly weighted integral $\int_{\Omega}(U V / r) d r d z$. The latter is computed by the Gauss quadratures for integrals with rational function weights (cf. [17] and [18]).

\section{Results and Discussion}

Figures 4-6 show a comparison between the numerical and analytical solutions. The latter is valid for $\mathrm{Na} \ll 1$ obtained in [11]. Plots of $\Theta$ along the horizontal line $z=0.5$ for $N a=1$ are shown in Figure 4. We can see the numerical solution is a little bigger at the boundary layer. The same curves are plotted in Figure 5 for $N a=0.1$. This time the two solutions are very close. Figure 6 compares the numerical solution with the analytical solution on $r=1 / 2$ when $\alpha=0.1$ and $\mathrm{Na}=1.0$. The discrepancy in the plots is however not surprising since the analytical results are valid only when $\mathrm{Na}<<1$. The surprise is that the analytical solution is quite good even for $\mathrm{Na}=1$. 


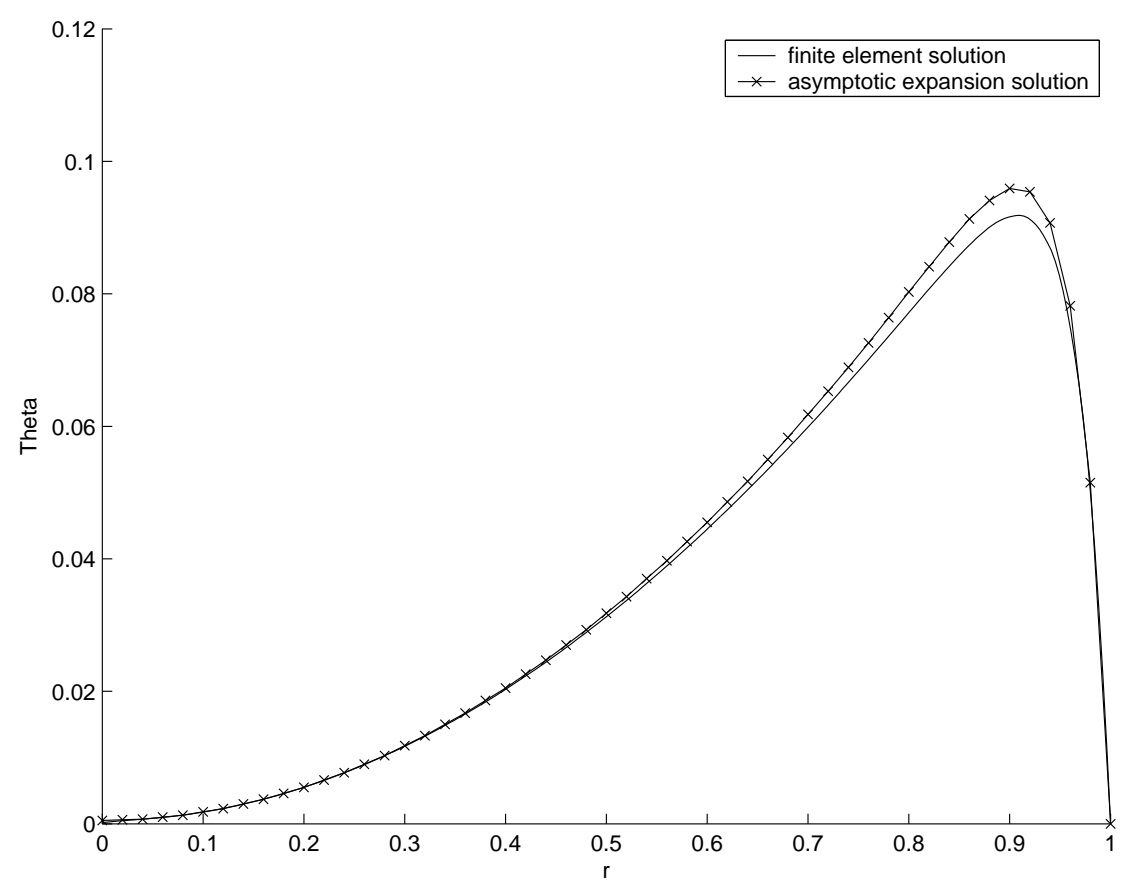

Figure 4: Comparison between numerical and analytical results for the non-isothermal temperature profile $\Theta$ at $z=.5$, for $\alpha=0.1$ and $\mathrm{Na}=1$

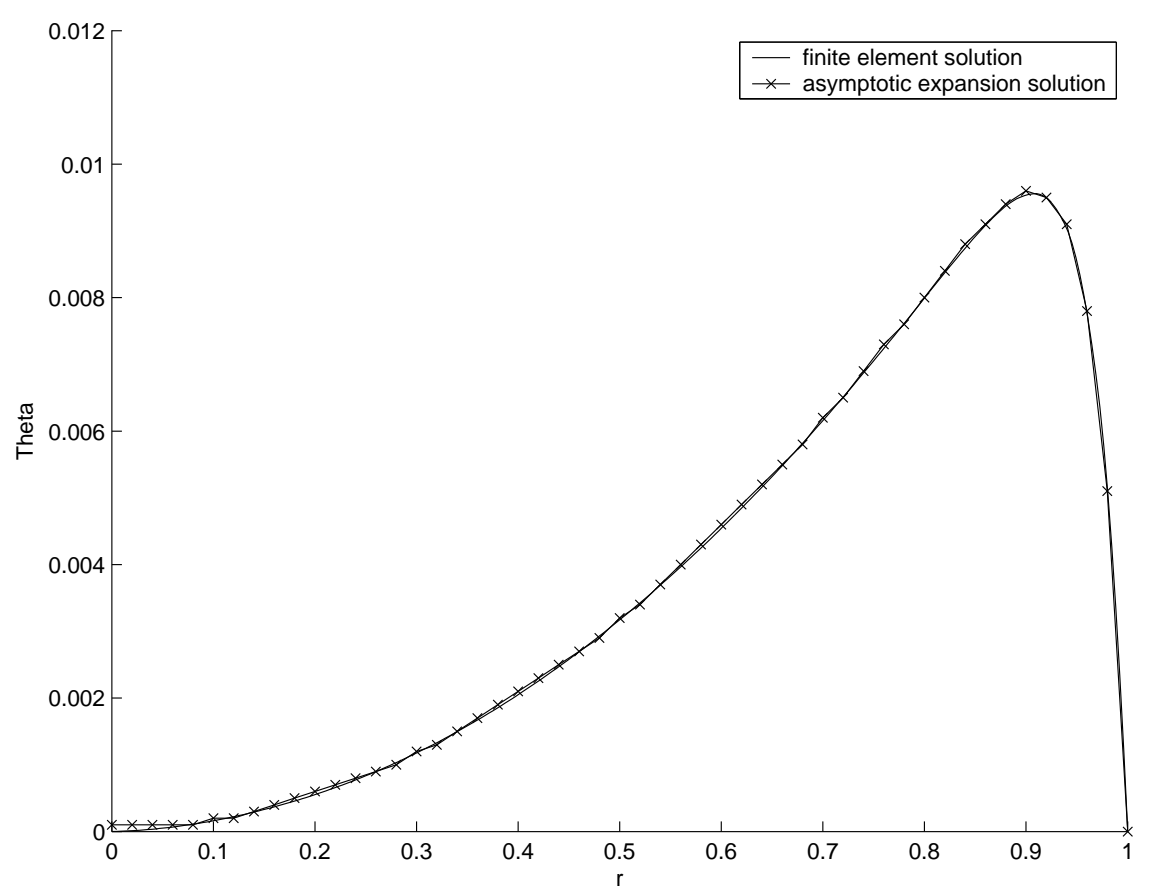

Figure 5: Comparison between numerical and analytical results for the non-isothermal temperature profile $\Theta$ at $z=.5$, for $\alpha=0.1$ and $\mathrm{Na}=0.1$ 


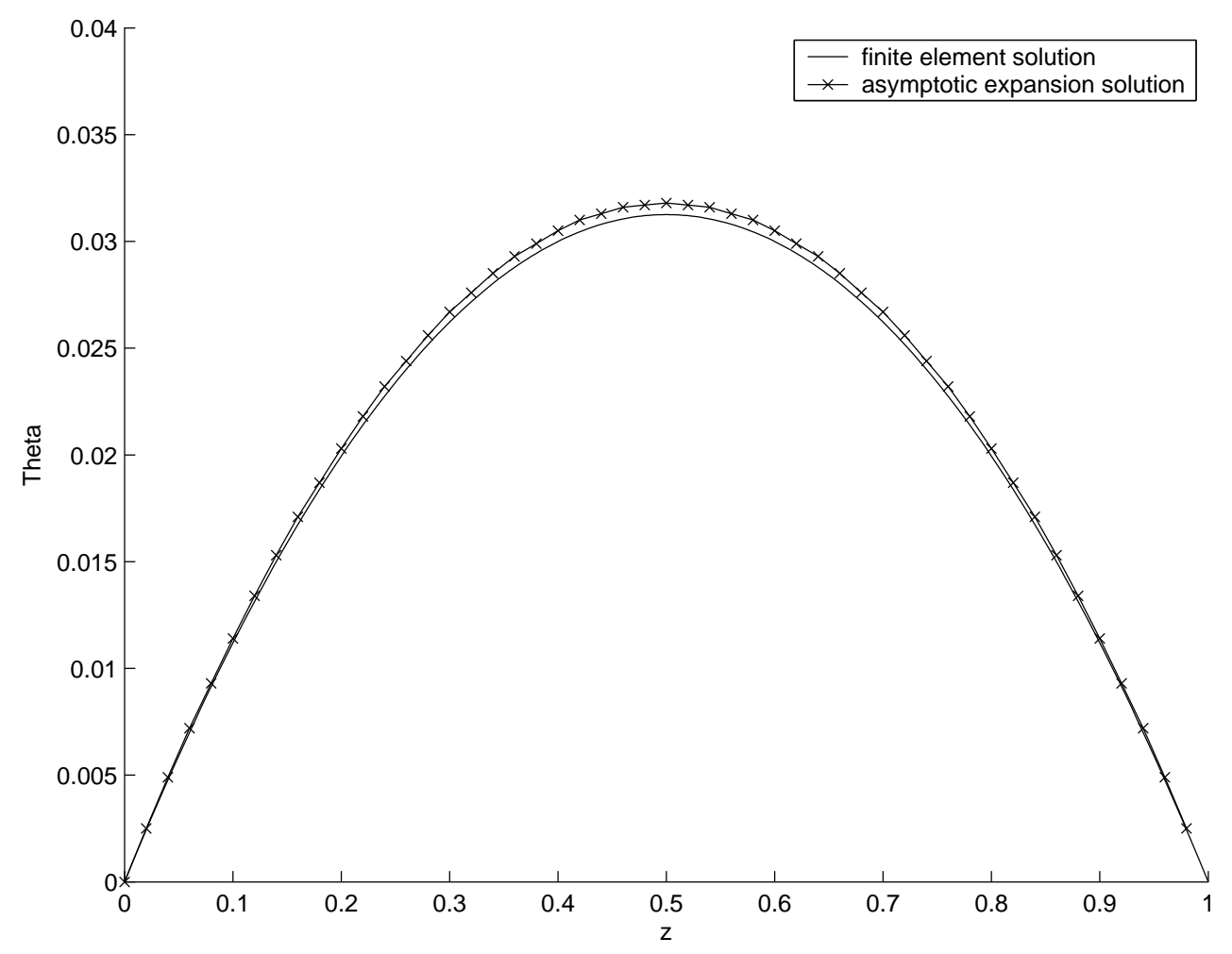

Figure 6: Comparison between numerical and analytical results for the non-isothermal temperature profile $\Theta$ at $r=.5$, for $\alpha=0.1$ and $\mathrm{Na}=1$

In viscometry, the flow confined to the gap between parallel plates is used to determine the viscosity of a fluid by measuring the torque exerted by the fluid on the stationary plate. If $\tilde{\mathcal{T}}$ is the torque then a dimensionless torque is given by the integral:

$$
\mathcal{T}=\frac{\tilde{\mathcal{T}}}{\left(2 \pi a^{4} \eta \omega / h\right)}=\left.\int_{0}^{1} r^{2} \frac{\partial W}{\partial z}\right|_{z=0} d r
$$

We plot the data $\mathcal{T}$ with various $\alpha$ (one for each curve) and $N a$ in Figure 7. For each $\alpha$ the torque decreases with $\mathrm{Na}$ while for a fixed $\mathrm{Na}$ the torque increases with aspect ratio $\alpha$. 


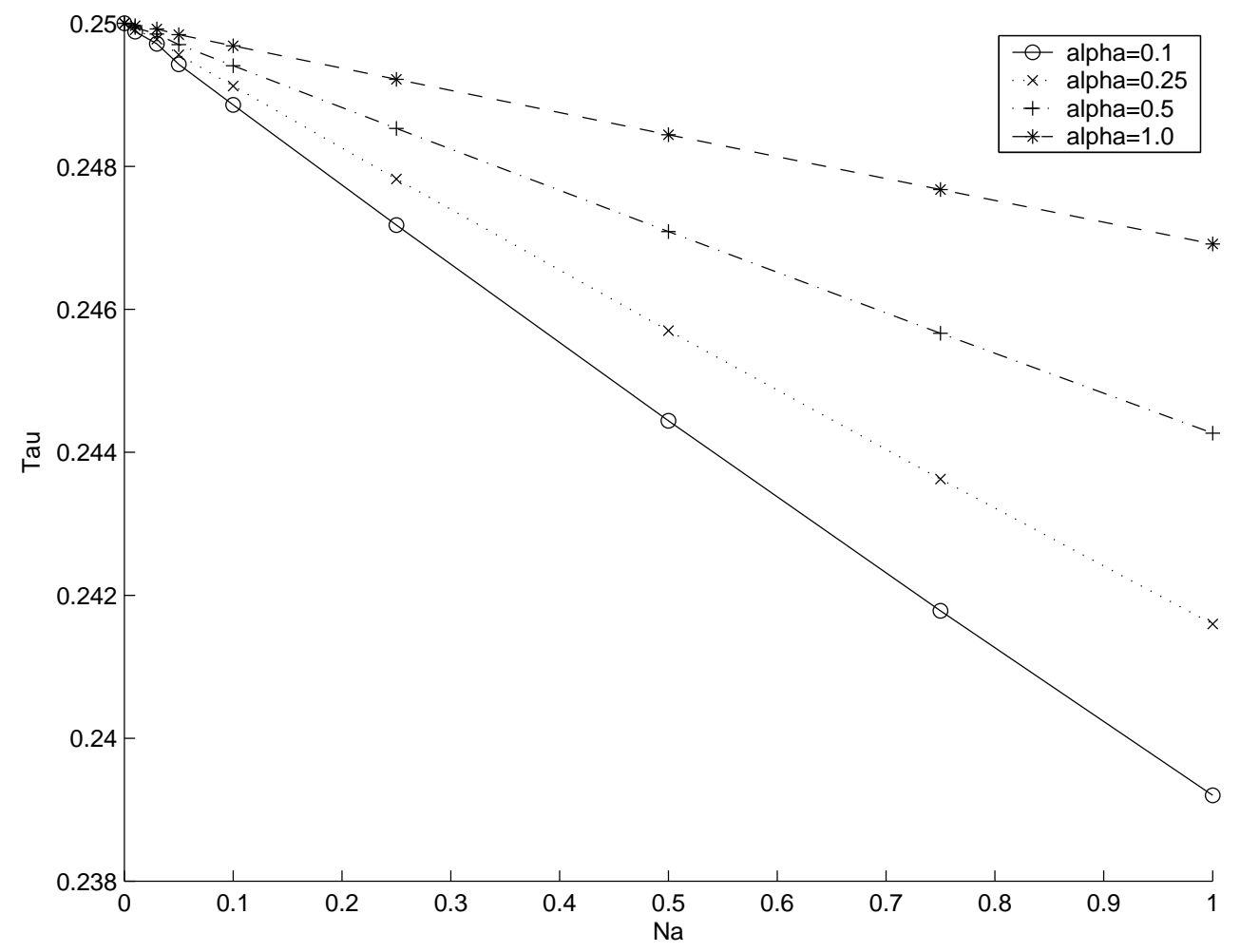

Figure 7: Numerically computed $\mathcal{T}$ against $\mathrm{Na}$ 


\begin{tabular}{||c||c|c||c|c||c||}
\hline \multicolumn{1}{||c||}{} & \multicolumn{2}{c||}{ Finite Element } & \multicolumn{2}{c||}{ Olagunju[11] } & $\begin{array}{c}\text { Plane } \\
\text { Couette } \\
\mathrm{Na}\end{array}$ \\
\cline { 2 - 5 } & $\alpha=0.1$ & $\alpha=1.0$ & $\alpha=0.1$ & $\alpha=1.0$ & \\
\hline 0 & 0.25000000 & 0.25000000 & 0.25000 & 0.25000 & 0.25000 \\
0.1 & 0.24886073 & 0.24968609 & 0.24885 & 0.24912 & 0.24862 \\
0.25 & 0.24717781 & 0.24921769 & 0.24713 & 0.24852 & 0.24659 \\
0.5 & 0.24444050 & 0.24844326 & 0.24427 & 0.24703 & 0.24330 \\
0.75 & 0.24178359 & 0.24767633 & 0.24140 & 0.24555 & 0.24013 \\
1.0 & 0.23920380 & 0.24691622 & 0.23854 & 0.24407 & 0.23707 \\
2.0 & 0.22958016 & 0.24394855 & 0.22707 & 0.24369 & 0.22578 \\
5.0 & 0.20596198 & 0.23567247 & 0.19268 & 0.23421 & 0.19892 \\
10.0 & 0.17787838 & 0.22362300 & 0.13537 & 0.21843 & 0.16829 \\
15.0 & 0.15799009 & 0.21329308 & 0.07085 & 0.20264 & 0.14730 \\
20.0 & 0.14298140 & 0.20429849 & 0.02074 & 0.18685 & 0.13179 \\
\hline
\end{tabular}

Table 1: Dimensionless torque on the bottom plate for $\alpha=0.1, \alpha=1.0$, and selected values of Na.

In Table 1, we give values of the dimensionless torque for $\alpha=0.1, \alpha=1.0$, and selected values of $\mathrm{Na}$ obtained from our numerical computation, and the analytical results in [11]. For comparison we have also included results for plane Couette flow [3]. All three results show good agreement for $\mathrm{Na} \leq 1$. For $\mathrm{Na}>1$ the agreement between the finite element and plane Couette solutions remain quite good with a maximum error of about $8 \%$ when $\mathrm{Na}=20$.

Table 1 also gives the calculated torque from the finite element solution and the solution of Olagunju [11] for $\alpha=1.0$. Again the agreement is quite good for $\mathrm{Na} \leq 1$. For large $\mathrm{Na}$ the agreement is not so good. The error for $\mathrm{Na}=20$ is about $8 \%$ but that is hardly surprising since the latter solution is only a linear approximation in Na. Although the plane Couette solution is valid for all values of $\mathrm{Na}$ the agreement with the finite element solution is poor with a difference of about $4 \%$ when $\mathrm{Na}=1.0$ to as much as $35 \%$ when $\mathrm{Na}=20$. Again this is not surprising since the plane Couette solution is valid only when $\alpha=0$.

\section{Conclusion}

The effect of viscous heating on the flow of a viscous fluid between two finite coaxial disks has been studied. By employing a finite element method we were able to solve the problem over a wide range of aspect ratio $\alpha$ and Nahme-Griffith number Na. These results have been compared to analytical solutions from asymptotic analyses. For small values of $\alpha$ and $\mathrm{Na}$ the agreement with asymptotic results are excellent. However, for $\alpha=O(1)$ and $\mathrm{Na}$ large the asymptotic results deviate significantly from the numerical solution. Asymptotic results showed a difference of as much as $35 \%$ in the torque on the stationary plate when $\alpha=1$ and $\mathrm{Na}=20$. This may be very important in certain applications such as viscometry. 


\section{References}

[1] R. B. Bird, R. C. Armstrong, and O. Hassager, Dynamics of Polymeric Liquids Vol 1, (1987), John Wiley and Sons.

[2] R. B. Bird, and R. M. Turian, Viscous heating effects in a cone and plate viscometer, Chem Engn Sc. 17 (1963) pp 331-334.

[3] R. M. Turian and R. B. Bird, Viscous heating in the cone-and-plate viscometer-II, Newtonian fluids with temperature-dependent viscosity and thermal conductivity, Chem Engn Sc, 18 (1963) pp 689-696.

[4] R. M. Turian, Viscous heating in the cone-and-plate viscometer-III, Non-Newtonian fluids with temperature-dependent viscosity and thermal conductivity, Chem Engn Sc, 20 (1965) pp 771-781.

[5] E. A. Kearsley, The viscous heating correction for viscometric flow, Trans Soc Rheol., 6 (1962) pp 253.

[6] R. Nahme, Ing Arch, 11 (1940) pp 191-209.

[7] J. M. White and S. J. Muller, Viscous heating and the stability of Newtonian and viscoelastic Taylor-Couette flows, Phys Rev Lett., 84 (22) (2000) pp 5130-5133.

[8] J. P. Rothstein and G. H. McKinley, Non-isothermal modification of purely elastic flow instabilities in torsional flow of polymeric fluids, Physics Fluids, 13 (2) (2001), pp 382-396.

[9] D. O. Olagunju, L. P. Cook, and G. H. McKinley, Effect of viscous heating on linear stability of viscoelastic cone-and-plate flow: axisymmetric case, J. Non-Newtonian Fluid Mech., 102 (2002) pp 321-342.

[10] J. Gavis and R. L. Laurence, Viscous heating in plane and circular flow between moving surfaces, Ind. Eng. Chem. Fundamentals, 7 (1968) 232-239.

[11] D. O. Olagunju, Analytical solutions for non-isothermal viscoelastic torsional flow in a bounded domain, J. Non-Newt. Fluid Mech., 112 (2003) 85-100.

[12] B. Heinrich, The Fourier-finite-element method for Poisson's equation in axisymmetric domains with edges, SIAM J. Numer. Anal., 33 (1996) pp 1885-1911.

[13] S. Börm and R. Hiptmair, Analysis of tensor product multigrid, Numer. Alg., 26 (2001) pp 331-356.

[14] S. Börm and R. Hiptmair, Multigrid computation of axisymmetric electromagnetic fields, Adv. in Comp. Math., 16 (2002) pp 331-356.

[15] J. Copalakrishnan and J. E. Pasciak, The convergence of V-cycle multigrid algorithms for axisymmetric Laplace and Maxwell equations, Department of Mathematics, Texas A\&M University, 2004, preprint.

[16] R. E. Bank and T. Dupont, An optimal order process for solving finite element equations, Math. Comp., 36(1981), pp 35-51. 
[17] T. E. Price, Numerically exact integration of a family of axisymmetric finite elements, Commun. Numer. Meth. Engng., 19 (2003) pp 253-261.

[18] J. D. Clayton and J. J. Rencis, Numerical integration in the axisymmetric finite element formulation, Adv. Engng. Soft., 31 (2001) pp 137-141. 